\title{
Energy-Efficient Routing Protocols in Wireless Sensor Networks: A Survey
}

\author{
Lovepreet Kaur \\ Student Mtech CSE $3^{\text {rd }}$ SEM \\ GNDU Reg. Jalandhar \\ Guide: Jyoteesh Malhotra, Ph.D (HOD)
}

\begin{abstract}
This paper represents energy efficient routing protocols in WSN. It is a collection of sensor nodes with a set of limited Processor and limited memory unit embedded in it. Reliable routing of packets from the sensor node to its base station is the most important task for the networks. The routing protocols applied for the other networks cannot be used here due to its battery powered nodes This paper gives an overview of the different routing strategies used in wireless sensor networks and gives a brief working model of energy efficient routing protocols in WSN. It also shows the comparison of these different routing protocols based on metrics such as mobility support, stability, issues and latency.
\end{abstract}

\section{Keywords}

Wireless sensor networks, mobility, energy efficiency, Leach, Issues in WSN, Routing protocols.

\section{INTRODUCTION}

Wireless sensor network is basically used to monitor environment. In WSN sensor node basically sense data, collect data from other nodes then process that data and then transmit this collected data to the base station. It is proved that node require much power or energy to transmit data rather then sensing as in case of WSN node has limited power and memory so the main concern is to save power to increase the life of sensor network. So in WSN energy efficient routing is essential due to limited power/battery .Routing protocols used in Sensor network are different from other networks routing protocols. Sensor networks are used in many applications like environment monitoring, health, industrial control units, military applications and in the various computing environments. Since the entire sensor nodes are battery powered devices, energy consumption of nodes during transmission or reception of packets affects the life-time of the entire network. To increase life time of sensor network number of protocols like LEACH and PEGASIS were developed and they show good progress then the previous routing protocols but still these are used for only static sensor nodes. This paper describes the existing routing strategies in WSN and section II defines various routing protocols gives an overview about energy efficient routing protocols like LEACH, HEED, DECA and PEGASIS.

\section{ROUTING MANIFESTATION}

This paper is organised as follows: In this section we define various routing protocols; section III gives overview of related work in this field and section IV discuss various issues and challenges in routing protocols.

All the proposed protocols will fall under any of the three categories: 1) Direct approach 2) Location based routing 3) Attribute based routing.

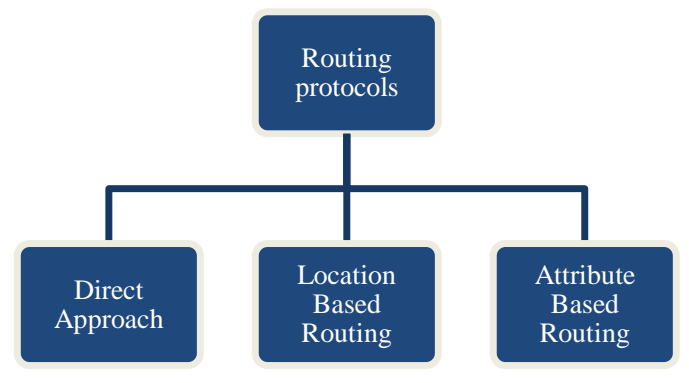

Fig .1: Protocol Structure

The simple flooding type routing protocols will be coming under the direct approach. It is quite simple in implementation but not energy efficient protocol. In the Location based nodes are addressed by their location. Location is achieved either through signal strength of nodes or through GPS(Global Positioning System)receivers in the network, in this technique some nodes should go to sleep mode if they are not used in current activity hence energy is saved and life time of sensor network increased.

In WSN instead of collecting information from all the nodes the application needs the data only from the nodes which satisfies its interest and this information gathering technique is widely called as the data centric approach or attribute based routing. Direct diffusion and rumour routing are the best examples for the attribute based routing or data centric approach.

\subsubsection{Location based routing:}

The routing of data to the nodes is done by the geographic location of the nodes (i.e.) nodes are identified by its location only. The location information of the individual nodes is obtained by the low power GPS receivers embedded in the nodes. Some of the most important protocols coming under the, Location based routing strategy are

- Greedy approach

- Compass routing

- DREAM

- GPSR

- GEAR

In the above mentioned protocols the first two follows the single path approach and next three follows the multi -path or flooding mechanism.

\subsubsection{Greedy approach:}

In [8] this packet travels from source to destination node through the set of intermediate nodes.

\subsubsection{Compass routing:}

In [9] this mechanism source node calculate the path cost i.e. 
distance from source to destination through different intermediate nodes and finally send packet to the path which has least cost in terms of energy saving.

\subsubsection{DREAM [A distance Routing Effect Algorithm for Mobility]}

In [10] this model tangent is drawn from source node to destination and packet is flooded to limited number of nodes which comes under the tangent range. it is a better one than its predecessors.

\subsubsection{GPSR [Greedy Perimeter Stateless Routing]}

The modified version of greedy-face-greedy algorithm is the Greedy perimeter stateless routing.

\subsubsection{GEAR [Geographic \& Energy Aware Routing]}

Y.Yu.et.Al [13] stated that GEAR uses the GIS (Geographical Information System) for finding location of sensor nodes in the network. GEAR limits the number of interests in Directed Diffusion by considering only a certain region rather than sending the interests to the whole network. The key idea to restrict the number of interests in directed diffusion by only considering a certain region rather than sending the interests to the whole network. GEAR thus complements Directed Diffusion and conserves more energy.

\subsubsection{GAF [Geographic Adaptive Fidelity]}

$\mathrm{Y} . \mathrm{Xu}, \mathrm{D}$.Estrin et.al [14] proposed that GAF conserves energy by turning off unnecessary nodes in the network without affecting the level of routing fidelity. It forms a virtual grid for the covered area. Each node uses its GPS-indicated location to associate itself with a point in the virtual grid. Nodes associated with the same point on the grid are considered equivalent in terms of the cost of packet routing. In order to balance the load the nodes change their states nodes in the same grid will go to the sleeping state to avoid unnecessary energy depletion.

\subsubsection{Data centric routing}

In location based routing node is selected on the basis of address of that node but in case of data centric (attribute based) nodes are separated on the basis of their work/content. Data is requested through queries, attribute-based naming is necessary to specify the properties of data. Some of the protocols which follow the data centric routing are,

- Directed diffusion

- SPIN

- Rumour routing

\subsubsection{Directed Diffusion}

In direct diffusion unnecessary operations of network layer routing is removed to save energy. A node that demands the data generates a request where an interest is specified according to the attribute-value based scheme defined by the application. The sink node issue interest to the network and each node check this "interest" on attribute-value based if interest matches data is sent back to sink on the basis of optimum gradient(select minimum cost route from source to sink to save energy) and if not matches then "interest" is passed to the neighbouring nodes.

\subsubsection{SPIN}

"Sensor Protocols for Information Negotiation" is the family of protocols based on data centric approach. It resolves flooding problem here the information is passed to the node which is in need of that information. In this model when node receive information it advertise to one hop neighbour node if these nodes require that data they get that information through REQ (request) packet and then the original data is sent to the one hop neighbour node. Hence redundancy of data or overlapping is removed in this protocol but if node which requires that information is not one hop neighbour of advertising node then there will be no delivery of data.

\subsubsection{Rumour routing}

It assumes bi-directional links between source and destination. It is used where number of events is less then number of queries i.e. for short transmission only. It maintains table of event-table in cache of node. When particular event occur node generate agent packet on the basis of that particular event and agent travel from one neighbour to other and on the other side sink i.e. base station send query for a particular event, agent select random path when both agent and query meet data is collected and deliver to sink/base station. If base station does not show interest in those events then its performance degrades and it is good for small event network if number of events increases then the cost of maintaining table increases. So it is good for small event network only.

\subsection{ENERGY EFFICIENCT ROUTING}

Energy efficiency is important in wireless sensor network because it directly affect the life of whole network, it is proved that in wireless network transmission of data consume more energy then data processing. Study on energy efficient routing in WSN brings this two broad classification of approaches. They are,

- Clustering approach

- Tree based approach

\subsubsection{Clustering techniques}

It is a great step for energy efficient routing. In this the nodes which are surplus of energy/battery i.e. node with enough energy can be used to process data and send the information while the low energy nodes are used for only sensing purpose i.e. to extend the life time of network. Its advantages are scalability, conserving communication bandwidth within the clusters, avoiding redundant message transfer between the sensor nodes and efficient communication. For this purpose network is divided into sub-units within each unit cluster head is assigned(node with enough energy) every other node in unit send data after sensing to only cluster head and cluster head will transmit to sink/base station. Some of the energy efficient routing protocols based on clustering are LEACH, HEED, DECA, etc

\subsubsection{LEACH}

Low energy adaptive clustering hierarchy it uses randomization for distributing the energy load among the sensors in the network. It assume base station is fixed and located far from the sensor nodes and the nodes are homogeneous and energy constrained. Here, one node called cluster-head $(\mathrm{CH})$ acts as the local base station. LEACH randomly rotates the high-energy cluster-head so that the activities are equally shared among the sensors and the sensors consume battery power equally. LEACH also performs data fusion, i.e. compression of data when data is sent from the clusters to the base station thus reducing energy dissipation and enhancing system lifetime. LEACH divides 
the total operation into rounds each round consisting of two phases: set-up phase and steady phase. In the set-up phase, clusters are formed and a $\mathrm{CH}$ is selected for each cluster. The $\mathrm{CH}$ is selected from the sensor nodes at a time with a certain probability. Here in the LEACH protocol multi cluster interference problem was solved by using unique CDMA codes for each cluster.

\subsubsection{HEED}

To overcome the disadvantage of LEACH protocol i.e. random selection of cluster head $\mathrm{CH}$ it is not equally distributed among all nodes in worst case it may randomly select only a certain node as $\mathrm{CH}$ which will depletes its energy and lifetime of network so to overcome this problem HEED was developed which selects the $\mathrm{CHs}$ based on both residual energy level and communication cost. Since HEED supports heterogeneous sensor nodes Emax may vary for different nodes according to its functionality and capacity. In this each node share its energy level and cost of communication with sink/base station through Emax level, $\mathrm{CHs}$ are selected with high Emax and low cost of communication.

\subsubsection{DECA}

DECA is an improved Distributed Efficient Clustering Approach. The basic difference between the HEED and DECA is how the nodes take the decision and the score computation. Basically new formula is used for calculation of remaining energy.

\subsubsection{Tree Based Approach}

Another approach is tree based in this technique network is assumed as tree like structure in which leaves nodes act as a source nodes and root node as a base station/sink. Source nodes sense data and then transfer each intermediate node aggregate data PEGASIS is example of such kind of technique.

\subsubsection{PEGASIS}

This method had been named as "Power Efficient Gathering in Sensor Information System". Each node communicates with neighbour node and takes turns transmitting to base station, thus energy is saved per round. In this approach chain is maintained either from source node to base station using some greedy algos or by base station which broadcast the chain to the entire nodes. Each node receives data from its immediate neighbour node and fuses this data to its own packet and sends to next neighbour. This protocol saves energy at various stages. First, in the local gathering, the distances that most of the nodes transmit are much less compared to transmitting to a cluster-head in LEACH. Second, the amount of data for the leader to receive is at most two messages instead of 20 (20 nodes per cluster in LEACH for a 100-node network). Finally, only one node transmits to the BS in each round of communication. But data is aggregated at each node so required level of information is not transmitted to base station in some applications.

\section{RELATED WORK}

In this section we discuss various researches in routing protocols in wireless sensor network.

B. Baranidharan [2] this paper proposed model Clustering based on k-means algorithm.

- Improved cluster head selection through RSS (Received Signal Strength) value.

- Alternate $\mathrm{CH}$ (Cluster Head) selection for continuous packet delivery.

- Shortest path to the super cluster further reduces the power consumption.

- Compression techniques for reduced data fusion cost.

.In [1] list routing model with the help of table from where each node gets shortest path from source to destination. In [4] paper, energy efficient routing protocols are classified into four main schemes: Network Structure, Communication Model, Topology Based and Reliable Routing. The routing protocols belonging to the first category can be further classified as flat or hierarchical. The routing protocols belonging to the second category can be further classified as Query-based or Coherent and non-coherent based or Negotiation-based. The routing protocols belonging to the third category can be further classified as Location-based or Mobile Agent-based.

The routing protocols belonging to the fourth category can be further classified as QoS-based or Multipath based. Then, an analytical survey on energy efficient routing protocols for WSNs is provided. In this paper, the classification initially proposed by Al-Karaki, is expanded, in order to enhance all the proposed papers since 2004 and to better describe which issues/operations in each protocol illustrate/enhance the energy.

Main goal of [5] is the latest progresses in WSN MAC protocol designs over the period 2002-2011. In the early development stages, designers were mostly concerned with energy efficiency because sensor nodes are usually limited in power supply. Recently, new protocols are being developed to provide multitask support and efficient delivery of busty traffic. Therefore, research attention has turned back to throughput and delay. This article details the evolution of WSN MAC protocols in four categories: asynchronous, synchronous, frame-slotted, and multichannel. These designs are evaluated in terms of energy efficiency, data delivery performance, and overhead needed to maintain a protocol's mechanisms. In [11] three routing protocols are discussed with issues and challenges.

\section{ISSUES AND CHALLENGES IN ROUTING}

Table 1: Performance Table 


\begin{tabular}{|c|c|c|c|c|c|c|}
\hline PROTOCOL & ISSUES & REVIEW & LATENCY & $\begin{array}{l}\text { Data } \\
\text { Security }\end{array}$ & $\begin{array}{l}\text { Data } \\
\text { aggregation }\end{array}$ & $\begin{array}{l}\text { Distributed } \\
\text { cluster head } \\
\text { and its stability } \\
\end{array}$ \\
\hline $\begin{array}{l}\text { Direct } \\
\text { Approach }\end{array}$ & $\begin{array}{l}\text { Not Energy } \\
\text { Efficient }\end{array}$ & $\begin{array}{l}\text { Simple in } \\
\text { Implementation But } \\
\text { only Flooding. }\end{array}$ & Low & No & No & N/A \\
\hline $\begin{array}{l}\text { Directed } \\
\text { Diffusion }\end{array}$ & $\begin{array}{l}\text { Query Based } \\
\text { model,Maching } \\
\text { require extra } \\
\text { energy, } \\
\text { Flooding of query } \\
\text { is unnecessary. }\end{array}$ & $\begin{array}{l}\text { Caching Overcome } \\
\text { delay and save } \\
\text { energy (save } \\
\text { different paths) }\end{array}$ & Higher & No & less & N/A \\
\hline $\begin{array}{l}\text { Rumour } \\
\text { Routing }\end{array}$ & $\begin{array}{l}\text { Maintaining Cost of } \\
\text { table and agent } \\
\text { high,Time to live } \\
\text { for query }\end{array}$ & $\begin{array}{l}\text { No Flooding of } \\
\text { query Good for } \\
\text { netwok which has } \\
\text { more events. }\end{array}$ & Acceptable & less & less & N/A \\
\hline LEACH & $\begin{array}{l}\text { Dynamic clustering } \\
\text { overhead }\end{array}$ & $\begin{array}{l}\text { Not applicable to } \\
\text { large NW multiple } \\
\text { nodes transmit to } \\
\text { Base Station }\end{array}$ & Acceptable & High & Yes & Limited \\
\hline HEED & $\begin{array}{l}\text { Energy loss for } \mathrm{CH} \\
\text { Selection Process }\end{array}$ & $\begin{array}{l}\text { Distribute Cluster } \\
\text { Head and Stability } \\
\text { is high. }\end{array}$ & Acceptable & High & Yes & Good \\
\hline PEGASIS & $\begin{array}{l}\text { Require Complete } \\
\text { Knowledge of } \\
\text { Network, } \\
\text { Bottelenek last } \\
\text { node, Delay is high }\end{array}$ & $\begin{array}{l}\text { No cluster head } \\
\text { problem,saves } \\
\text { energy but need to } \\
\text { avoid delay. }\end{array}$ & Higher & High & No & N/A \\
\hline
\end{tabular}

\section{OPEN ISSUES AND FUTURE SCOPE}

Routing in sensor network is a very attractive phase of wireless communication. This paper summarized recent research in data routing to save energy of sensor network and classified the approaches into three main categories, namely direct approach, attribute based and location-based.The table summarizes the various protocols in this paper. Data aggregation is a open issue in sensor network routing protocols in terms of energy saving and traffic optimization.

Protocols, which name the data and query the nodes based on some attributes of the data are categorized as data-centric or attribute based. Many of the researchers follow this paradigm in order to avoid the overhead of forming clusters, the use of specialized nodes etc. However, the naming schemes such as attribute-value pairs might not be sufficient for complex queries and they are usually dependent on the application. Efficient standard naming schemes are one of the most interesting future research direction related to this category.

Many routing protocols follow the creteria in which sensor network is integrated with wired network like in monitoring application need the data that is collected by sensor nodes and to be transmitted to the server for further classification.On the other hand,the requests from the user should be made to the sink through Internet. Since the routing requirements of each environment are different, further research is necessary for handling these kinds of situations.

And in case of cluster based routing protocols the selection of cluster head is challenge because sometimes those nodes are selected as a cluster head whose energy or battery level is less.A cluster-head performs aggregation of data and sends it to the sink on behalf of the nodes within its cluster. The most interesting research issue regarding such protocols is how to form the clusters so that the energy consumption and contemporary communication metrics such as latency are optimized. The factors affecting cluster formation and clusterhead communication are open issues for future research.

\section{CONCLUSION}

Unlike other networks, WSNs are designed for specific applications. Thus the existing routing strategies in the wireless sensor networks and their corresponding protocols had been explained. Though the protocol like LEACH, HEED, DECA, SPIN, and PEGASIS are proved to be energy efficient than its previous models the main pitfalls in these protocols are that nodes are assumed to be static and stationary.

This paper surveyed different categories of routing protocols to save energy and extend the life time of sensor network. We have summarized and compared different proposed designs, algorithms, protocols, and services. There are still many issues to be resolved around WSN applications such as communication architectures, security, and management. By solving these issues, we can close the gap between technology and application.

\section{REFERENCES}

[1]. B.P.S Sahoo, Satyajit Rath, Deepak Puthal "Energy Efficient Protocols for Wireless Sensor Networks: A Survey and Approach" International Journal of Computer Applications (0975 -8887) Volume 44 No18, April 2012.

[2]. B. Baranidharan Assistant Professor, School of computing, SASTRA University, Tamil Nadu, India“A Survey on Energy Efficient Protocols for Wireless Sensor Networks" International Journal of Computer Applications $(0975$ - 8887)Volume 11 - No.10, December 2010.

[3]. Forum Presentation by Eileen Balci, Stephanie Reese, and Shannon Seefeld. 
[4]. Nikolaos A. Pantazis, Stefanos A. Nikolidakis and Dimitrios D. Vergados, Senior Member, IEEE "EnergyEfficient Routing Protocols in Wireless Sensor Networks: A Survey"IEEE COMMUNICATIONS SURVEYS \& TUTORIALS, VOL. 15, NO. 2, SECOND QUARTER 2013.

[5]. Pei Huang, Li Xiao,Senior Member, IEEE,Soroor Soltani,Student Member, IEEE,Matt W. Mutka, and Ning $\mathrm{Xi}$, Fellow, IEEE, "The Evolution of MAC Protocols in Wireless Sensor Networks: A Survey" IEEE COMMUNICATIONS SURVEYS \& TUTORIALS, VOL. 15, NO. 1, FIRST QUARTER 2013.

[6]. Himangi Pande Department of Computer Science SGBA University, Amravati, India, Kalpana Saharan. "Various Ways to Implement Energy Efficient WiseMAC Protocol for Wireless Sensor Network" 2013 IEEE International Conference on Systems, Man, and Cybernetics.

[7]. VSRD International Journal of Computer Science \& Information Technology, Vol. IV Issue I January 2014 "EVALUATING THE GAPS OF DIFFERENT ENERGY EFFICIENT PROTOCOLS OF WSN" Daljeet Kaur Research Scholar, Department of Computer Science \& Engineering, S.B.S. Campus, Firozepur, Punjab, INDIA.

[8]. I. Stojmenovic and X. Lin. "GEDIR: Loop-Free Location Based Routing in Wireless Networks", In International Conference on Parallel and Distributed Computing and Systems, Boston, MA, USA, Nov. 3-6,
1999.

9]. E.Kranakis, H.Singh and J.Urrutis. "Compass routing on geometric networks. In proc. $11^{\text {th }}$ Canadian conference on Computational Geometry", Pages 51-54, Vancouver, August 1999.

[10]. S. Basagni and et. al. A Distance Routing Effect Algorithm for Mobility (DREAM). In ACM/IEEEInt. Conf. on Mobile Computing and Networking (MobiCom'98), October 1998.

[11]. Ms. Parul Tyagi, Ms. Surbhi Jain "Comparative Study of Routing Protocols in Wireless Sensor Network" International Journal of Advanced Research in Computer Science and Software Engineering Research Paper Volume 2, Issue 9, September 2012.

[12]. Kemal Akkaya, Mohamed Younis "Routing protocols for wireless sensor networks” Elsevier, sept 2003.

[13]. Y. Yu, D. Estrin, and R. Govindan, "Geographical and Energy-Aware Routing: A Recursive Data Dissemination Protocol for Wireless Sensor Networks," UCLA Computer Science Department Technical Report, UCLACSD TR-01-0023,May,2001,pp.1-11.

[14]. X. J. Heidemann, and D. Estrin, "Geography-informed energy conservation for ad hoc routing", in the Proceedings of the 7th Annual ACM/ IEEE International Conference on Mobile Computing and Networking (MobiCom) July 2001, Rome,Italy,pp.381677-381685. 